This article has been accepted for publication in MNRAS (c) 2018 The Authors. Published by Oxford University Press on behalf of the Royal Astronomical Society. All rights reserved. 


\title{
Cosmic degeneracies III: $N$-body simulations of interacting dark energy with non-Gaussian initial conditions
}

\author{
M. Hashim, ${ }^{1,2,3 \star}$ C. Giocoli ${ }^{\oplus},{ }^{1,2,4}$ M. Baldi ${ }^{\oplus,},{ }^{1,2,4}$ D. Bertacca $, 5,6,7,8$ and R. Maartens ${ }^{3,9}$ \\ ${ }^{1}$ Dipartimento di Fisica e Astronomia, Alma Mater Studiorum Università di Bologna, viale Berti Pichat, 6/2, I-40127 Bologna, Italy \\ ${ }^{2}$ INAF - Osservatorio Astronomico di Bologna, via Ranzani 1, I-40127 Bologna, Italy \\ ${ }^{3}$ Department of Physics \& Astronomy, University of the Western Cape, Cape Town 7535, South Africa \\ ${ }^{4}$ INFN - Sezione di Bologna, viale Berti Pichat 6/2, I-40127 Bologna, Italy \\ ${ }^{5}$ Dipartimento di Fisica e Astronomia 'G. Galilei', Università degli Studi di Padova, via Marzolo 8, I-35131 Padova, Italy \\ ${ }^{6}$ INFN, Sezione di Padova, via Marzolo 8, I-35131 Padova, Italy \\ 7 Argelander-Institut für Astronomie, Auf dem Hugel 71, D-53121 Bonn, Germany \\ ${ }^{8}$ Departament de Física Quàntica i Astrofisíca \& Institut de Ciències del Cosmos, Universitat de Barcelona, Martí i Franquès 1, E-08028 Barcelona, Spain \\ ${ }^{9}$ Institute of Cosmology \& Gravitation, University of Portsmouth, Portsmouth PO1 3FX, UK
}

Accepted 2018 September 5. Received 2018 August 22; in original form 2018 June 12

\begin{abstract}
We perform for the first time $N$-body simulations of interacting dark energy assuming nonGaussian initial conditions, with the aim of investigating possible degeneracies of these two theoretically independent phenomena in different observational probes. We focus on the largescale matter distribution, as well as on the statistical and structural properties of collapsed haloes and cosmic voids. On very large scales, we show that it is possible to choose the interaction and non-Gaussian parameters such that their effects on the halo power spectrum cancel, and the power spectrum is indistinguishable from a $\Lambda$ cold dark matter $(\Lambda \mathrm{CDM})$ model. On small scales, measurements of the non-linear matter power spectrum, halo-matter bias, halo and subhalo mass function, and cosmic void number function validate the degeneracy determined on large scales. However, the internal structural properties of haloes and cosmic voids, namely halo concentration-mass relation and void density profile, are very different from those measured in the $\Lambda \mathrm{CDM}$ model, thereby breaking the degeneracy. In practice, the values of $f_{\mathrm{NL}}$ required to cancel the effect of interaction are already ruled by observations. Our results show in principle that the combination of large- and small-scale probes is needed to constrain interacting dark energy and primordial non-Gaussianity separately.
\end{abstract}

Key words: galaxies: formation-dark energy-dark matter-large-scale structure of Universe-cosmology: theory.

\section{INTRODUCTION}

According to the most recent measurements of cosmic microwave background (CMB) anisotropies performed by the Planck satellite mission (Ade et al. 2016a), the standard $\Lambda$ cold dark matter $(\Lambda \mathrm{CDM})$ cosmological model is still extremely successful in reproducing different observational data sets. This in turn favours the more economic cosmological constant $\Lambda$ as an explanation of the late-time cosmic acceleration over alternative and more complex dark energy (DE) or modified gravity (MG) models. None the less, theoretical problems in understanding the energy scale and the time evolution of $\Lambda$ (known as the fine-tuning and coincidence problems, respectively, see e.g. Weinberg 1989; Padilla 2015) as well as recent observational tensions between $\mathrm{CMB}$ cosmological constraints

^E-mail: mahmoudyousif.hashim@unibo.it and those inferred from independent probes in the local Universe (see e.g. Vikhlinin et al. 2009; Heymans et al. 2013; Ade et al. 2016e; Simpson et al. 2016; Hildebrandt et al. 2017) motivate the investigation of such alternative and more complex scenarios.

In particular, various possible realizations of interacting dark energy (IDE) models (see e.g. Wetterich 1995; Amendola 2000; Amendola, Baldi \& Wetterich 2008; Pettorino \& Baccigalupi 2008; Baldi 2011a, 2012c; Pourtsidou, Skordis \& Copeland 2013) based on a direct energy-momentum exchange between a DE scalar field and the CDM particle sector, have attracted significant interest and for small values of the interaction strength appear still consistent with current CMB constraints (Salvatelli et al. 2013; Costa et al. 2014; Salvatelli et al. 2014; Ade et al. 2016b).

Similarly, measurements of higher order statistics of the CMB anisotropies are consistent with a nearly Gaussian distribution of the primordial curvature perturbations by providing very tight 
constraints on the primordial non-Gaussianity (hereafter PNG) parameters: $f_{\mathrm{NL}}^{\text {loc }}=0.5 \pm 5.0$ and $f_{\mathrm{NL}}^{\mathrm{eq}}=-4.0 \pm 43.0$ for the local and equilateral configurations, respectively (Ade et al. 2016c). As some level of non-Gaussianity in the primordial density distribution is a common and clean prediction of basically all models of inflation (see e.g. Maldacena 2003) - i.e. the hypothetical mechanism driving the early exponential expansion of the Universe - measurements of PNG are considered as a smoking gun to discriminate between various inflationary models (Bartolo et al. 2004; Giannantonio et al. 2014).

Despite the tight constraints on the PNG amplitude from Planck data, large-scale structure (LSS) observations in the late universe coming from the next generation of wide-field galaxy redshift surveys could outperform these constraints. More specifically, recent measurements of galaxy clustering and of the integrated SachsWolfe (ISW) effect already provide constraints of $\sigma\left(f_{\mathrm{NL}}^{\text {loc }}\right) \sim 30$ (Ross et al. 2013; Giannantonio et al. 2014; Leistedt, Peiris \& Roth 2014), while future redshift galaxy surveys like EUCLID (Laureijs et al. 2011) and SKA (Camera, Santos \& Maartens 2015; Maartens et al. 2015) are forecast to outperform the CMB in constraining PNG, especially via the multitracer method (Alonso \& Ferreira 2015; Fonseca et al. 2015).

This is possible due to the various observational signatures that PNG imprints on LSS at late times, namely on the abundance of massive objects (which can be either enhanced or suppressed for positive and negative values of the PNG amplitude, respectively), on the bias between galaxies and the underlying matter distribution (that becomes scale dependent on large scales in the presence of some PNG) and on the 3-point correlation function of galaxies that encodes the shape of PNG (for more details, see e.g. Desjacques \& Seljak 2010; Liguori et al. 2010; Desjacques, Jeong \& Schmidt 2018).

Recent studies on the effects of IDE models on structure formation (Baldi et al. 2010; Cui, Baldi \& Borgani 2012; Hashim, Bertacca \& Maartens 2014; Moresco et al. 2014; Duniya, Bertacca \& Maartens 2015) have shown that similar features may arise also in these models. In particular, Hashim et al. (2014) showed that the observational signatures of IDE and PNG on the large-scale galaxy power spectrum can mimic each other. This is due to the fact that some models of IDE introduce a $k^{-2}$ scale dependence in the matter density contrast on very large scales, mimicking PNG scale-dependent halo bias. Also on non-linear scales, numerical simulations of IDE (see e.g. Baldi \& Pettorino 2011; Baldi 2012b,a; Cui et al. 2012) and of PNG (Grossi et al. 2007; Pillepich, Porciani \& Hahn 2010; Wagner, Verde \& Boubekeur 2010; LoVerde \& Smith 2011) scenarios showed that IDE enhances the abundance of massive haloes in a similar way to PNG with a positive amplitude.

This degenerate behaviour between PNG and IDE indicates that separate observational constraints on the PNG amplitude and the IDE interaction rate could be misinterpreted or possibly that their joint effects could become indistinguishable from the standard $\Lambda \mathrm{CDM}$ reference model. This represents the main motivation for the present work, where we will present for the first time a joint numerical analysis of non-linear structures forming from PNG initial conditions through an IDE cosmological evolution. Our main goal is to test whether such degeneracy holds for all observables at all scales and if not to identify specific statistics that clearly disentangle the two phenomena. To this end we will consider - as a proof of concept - very large values of the PNG amplitude $f_{\mathrm{NL}} \approx \mathcal{O}(100)$ which are already ruled out by CMB observations for the simple case of scale-independent non-Gaussianity. This allows us to obtain larger effects on structure formation and to identify more clearly the degeneracy with DE interactions. Although not directly applicable to realistic PNG scenarios for the case of a scale-independent $f_{\mathrm{NL}}$, our results will provide a guideline for scale-dependent PNG models (see e.g. Sefusatti et al. 2009; Oppizzi et al. 2018) having $f_{\mathrm{NL}} \approx \mathcal{O}(100)$ or larger at the scales relevant for non-linear structure formation while remaining consistent with $\mathrm{CMB}$ constraints at the Planck pivot scale.

This paper is organized as follows: in Section 2 we introduce IDE and PNG extensions to the standard $\Lambda \mathrm{CDM}$ scenario. In Section 3, we use the linear halo power spectrum as an observational probe to test the IDE-PNG degeneracy on large scales. In Section 4, we test the IDE-PNG degeneracy on non-linear scales by running a set of $N$ body simulations for all models under consideration. In Section 5, we present all results for the non-linear matter power spectrum, halo-matter bias, halo mass function, subhalo mass function, halo concentration-mass relation, void number density, and void density profiles. Finally, conclusions are summarized in Section 6.

\section{NON-STANDARD COSMOLOGICAL MODELS}

In this section, we present the two non-standard extensions to the fiducial $\Lambda \mathrm{CDM}$ model that we will consider in this work. The first extension is based on the assumption of a non-Gravitational interaction between CDM particles and a dynamical DE scalar field. The other extension relays on a non-Gaussian distribution of the primordial density field as generically predicted by inflationary models.

\subsection{Interacting dark energy}

Various models of IDE have been proposed in the literature over the past two decades (see e.g. Amendola 2000, 2004; Koyama, Maartens \& Song 2009; Baldi 2011a; Clemson et al. 2012). In this paper, we consider the most widely studied example of such models based on a quintessence dynamical scalar field $\phi$ playing the role of the DE, subject to a self-interaction potential $V(\phi)$ and to a direct interaction with the CDM fluid via energy-momentum exchange (Amendola 2000; Bertolami \& Martins 2000). The background evolution of such cosmological scenarios is governed by the KleinGordon equation for the scalar field:

$\ddot{\phi}+3 H \dot{\phi}+\frac{d V}{d \phi}=\sqrt{\frac{2}{3}} \kappa \beta \rho_{c}$,

and by the continuity equations of the different components that contribute to the total energy density of the universe:

$$
\begin{aligned}
& \dot{\rho}_{\mathrm{c}}+3 H \rho_{\mathrm{c}}=-\sqrt{\frac{2}{3}} \kappa \beta \rho_{\mathrm{c}} \dot{\phi}, \\
& \dot{\rho}_{\mathrm{b}}+3 H \rho_{\mathrm{b}}=0, \\
& \dot{\rho}_{\mathrm{r}}+4 H \rho_{\mathrm{r}}=0,
\end{aligned}
$$

as well as by the Friedmann constraint

$3 H^{2}=\kappa^{2}\left(\rho_{\phi}+\rho_{\mathrm{c}}+\rho_{\mathrm{b}}+\rho_{\mathrm{r}}\right)$,

where $\rho_{\mathrm{c}}, \rho_{\mathrm{b}}$, and $\rho_{r}$ are the energy density of CDM, baryons, and radiation, respectively. An overdot represents a derivative with respect to the cosmological time $t$. The Hubble function is defined as $H \equiv \dot{a} / a$ where $a$ is the scale factor and $\kappa^{2}=8 \pi G$. The parameter $\rho_{\phi}$ represents the energy density of the DE fluid defined as $\rho_{\phi}=\dot{\phi}^{2} / 2+V(\phi)$. The right-hand side source terms in equations (1) and (2) represent the interaction parameter between CDM 
particles and DE that is proportional to the CDM energy density $\rho_{\mathrm{c}}$ through the dimensionless constant $\beta$ that sets the strength of the coupling. The sign of the $\beta \dot{\phi}$ term determines the direction of the energy-momentum exchange between the two interacting components. In order to fulfil Bianchi identities and not violate total energy-momentum conservation, the source terms in equations (1) and (2) should be equal and have opposite sign.

By integrating the CDM conservation equation (2) one gets the time evolution of the CDM density as

$\frac{\rho_{\mathrm{c}}}{\rho_{\mathrm{c} 0}}=a^{-3} \exp \left(-\sqrt{\frac{2}{3}} \kappa \beta \phi\right)$,

which shows a basic property of IDE models: matter density is not separately conserved as the energy exchange results in a timedependent CDM particle mass. In this work, we consider the exponential form for the self-interaction potential (Lucchin \& Matarrese 1985; Wetterich 1988),

$V(\phi)=A \exp \left(-\sqrt{\frac{2}{3}} \kappa \lambda \phi\right)$,

where $A$ and $\lambda$ are constants.

In the Newtonian gauge, the perturbed metric (assuming flatness and vanishing anisotropic stress) is given by

$\mathrm{d} s^{2}=\left[-(1+2 \Phi) \mathrm{d} t^{2}+(1-2 \Phi) a^{2} \mathrm{~d} x^{2}\right]$,

where $\Phi$ is the gravitational potential. The Poisson equation is ${ }^{1}$ (Hashim et al. 2014):

$\nabla^{2} \Phi=\frac{\kappa^{2}}{2}\left[\rho_{\mathrm{c}} \Delta_{\mathrm{c}}+\rho_{\phi} \Delta_{\phi}-\sqrt{\frac{2}{3}} \kappa \beta \dot{\phi} \frac{\rho_{\mathrm{c}}}{\left(\rho_{c}+\rho_{\phi}\right)}\left(v_{\phi}-v_{\mathrm{c}}\right)\right]$,

where $\Delta_{\mathrm{c}, \phi}$ are the comoving density contrasts and $v_{\mathrm{c}, \phi}$ are the velocity potentials, defined by $\boldsymbol{v}_{\mathrm{c}, \phi}=\nabla v_{\mathrm{c}, \phi}$, so that $\theta_{\mathrm{c}, \phi}=-k^{2} v_{\mathrm{c}, \phi}$, where $\theta$ is the velocity divergence. The velocity potentials include a $k^{-2}$ scale dependence due to the potential $\Phi$ in the Euler equation see equation (10) below. Therefore, the coupling term in the Poisson equation (9) introduces a $k^{-2}$ scale dependence to the matter growth factor on large scales. Since $\Delta_{c, \phi}$ are gauge-invariant, the resulting $k^{-2}$ signal is an explicit coupling effect and not a false gauge effect.

The perturbed conservation equations are then given by (Hashim et al. 2014)

$$
\begin{aligned}
& \dot{v}_{i}+H v_{i}+\frac{c_{s i}^{2}}{\left(1+w_{i}\right)} \Delta_{i}+\Phi \\
& =\frac{1}{\left(1+w_{i}\right) \rho_{i}}\left[Q_{i}\left(v-v_{i}\right) \rho_{c}+f_{i}\right],
\end{aligned}
$$

$$
\begin{aligned}
& \dot{\Delta}_{i}-3 w_{i} H \Delta_{i}-k^{2}\left(1+w_{i}\right) v_{i}-\frac{9}{2} H^{2}\left(1+w_{i}\right)\left(1+w_{t}\right)\left(v_{i}-v\right) \\
& =\frac{\mathcal{Q}_{i}^{\Delta}}{H},
\end{aligned}
$$

where $Q_{\phi}=\sqrt{2 / 3} \kappa \beta \rho_{c} \dot{\phi}=-Q_{c}$ and $i$ indicates CDM and scalar field $\phi$, respectively, $c_{s i}$ is the sound-speed of the $i$ th species (which is vanishing for CDM while for DE perturbations $\left.c_{s \phi}=1\right), w_{t}$ is the total equation of state, $v=1 /\left(1+w_{t}\right) \sum_{i}\left(1+w_{i}\right) \Omega_{i} v_{i}$ is the total peculiar velocity potential, and $f_{i}$ is the momentum transfer potential given by (Koyama et al. 2009)

$f_{i}=Q_{i}\left(v_{\phi}-v\right)$.

\footnotetext{
${ }^{1}$ We also ignore baryons for simplicity.
}

The source term on the right-hand side of equation (11) is given by

$$
\begin{aligned}
\mathcal{Q}_{i}^{\Delta}= & \frac{Q_{i}}{\rho_{i}}\left[\frac{\dot{Q}_{i}}{Q_{i}}-\frac{\dot{\rho}_{i}}{\rho_{i}}\right] v_{i}-\frac{Q_{i}}{\rho_{i}}\left[3+\frac{Q_{i}}{\left(1+w_{i}\right) \rho_{i} H}\right]\left(v-v_{i}\right) \\
& -\frac{1}{\rho_{i}}\left[3+\frac{Q_{i}}{\left(1+w_{i}\right) \rho_{i} H}\right] f_{i}+\frac{Q_{i}}{\rho_{i}}\left[3\left(1+w_{i}\right)+\frac{Q_{i}}{\rho_{i} H}\right] v_{i} \\
& +\frac{1}{\rho_{i} H} \delta Q_{i}-\frac{Q_{i}}{\rho_{i} H}\left[\frac{c_{s i}^{2}}{\left(1+w_{i}\right)}+1\right] \Delta_{i}+2 \frac{Q_{i}}{\rho_{i} H} \Phi .
\end{aligned}
$$

These equations fully specify the evolution of the linear gaugeinvariant perturbations of the coupled system, we refer the interested reader to Hashim et al. (2014) for a more complete derivation of these equations.

As we will be interested in the evolution of the system at small scales and beyond the linear regime (see Section 4.1 for details), we also recall (see e.g. Amendola 2004) that in the Newtonian limit, used for the $\mathrm{N}$-body implementations, the evolution equation for CDM density perturbations, equations (10) and (11) imply:

$\ddot{\delta}_{c}+2 H\left(1-\beta \frac{\dot{\phi}}{H \sqrt{6}}\right) \dot{\delta}_{c}-\frac{\kappa^{2}}{2} \rho_{c}\left(1+\frac{4}{3} \beta^{2}\right) \delta_{c}=0$,

since comoving and Newtonian density contrasts are equal, i.e. $\Delta_{\mathrm{c}} \approx \delta_{\mathrm{c}}$, and we ignore derivatives of scalar field perturbations. The coupling terms in equation (14) are: $\beta \dot{\phi}$, which represents an extra friction arising as a consequence of momentum conservation, and $4 \beta^{2} / 3$, which is responsible for the fifth force acting on CDM perturbations.

\subsection{Primordial non-Gaussianity}

Local-type non-Gaussianity in the primordial curvature perturbations, that maximizes the bispectrum in the squeezed shape, is parametrized by

$\Phi=\Phi_{\mathrm{G}}+f_{\mathrm{NL}}^{\text {loc }}\left(\Phi_{\mathrm{G}}^{2}-\left\langle\Phi_{\mathrm{G}}^{2}\right\rangle\right)$,

where $\Phi_{\mathrm{G}}$ is the Gaussian gravitational field and $f_{\mathrm{NL}}^{\text {loc }}$ is the PNG parameter. Single-field inflation models predict a very small value of $f_{\mathrm{NL}}^{\text {loc }}$ (Maldacena 2003), but multifield models can generate large non-Gaussianity in squeezed configurations (Moroi \& Takahashi 2001; Lyth \& Wands 2002).

On large scales, PNG enhances the large peaks of matter perturbations (Matarrese, Verde \& Jimenez 2000; LoVerde et al. 2008; Matarrese \& Verde 2008). This introduces a scale-dependent signal in the bias between the virial collapsed objects at high peaks and the underlying traced matter. By measuring the cross halo-matter power spectrum $P_{\mathrm{mh}}$ in $N$-body simulations with local-type non-Gaussian initial conditions, many authors have confirmed that the large-scale bias is scale dependent (see e.g. Dalal et al. 2008; Pillepich et al. 2010):

$P_{\mathrm{hm}}(k, z)=\left[b_{\mathrm{G}}(z)+\Delta b(k, z)\right] P_{\mathrm{mm}}(k, z)$,

where $P_{m m}$ is the matter auto-power spectrum, $b_{\mathrm{G}}$ is the Gaussian bias and

$\Delta b(k, z)=3 f_{\mathrm{NL}}\left[b_{\mathrm{G}}(z)-1\right] \frac{\delta_{\text {crit }} \Omega_{m}}{D_{\mathrm{c}}(z) T(k)} \frac{H_{0}^{2}}{k^{2}}$,

with $\delta_{\text {crit }}$ being the critical overdensity for halo collapse, $T(k)$ the transfer function, and $D_{\mathrm{c}}$ the linear dark matter growth factor which is normalized to $a$ in the matter dominated era. On very large scales, $T \rightarrow 1$ and so $\Delta b \circ f_{\mathrm{NL}} k^{-2}$. Since we only consider local-type PNG in the current analysis, for simplicity we drop the loc superscript from our notation. 
Table 1. The cosmological parameters used in this paper, consistent with the WMAP7 CMB data best fit (Komatsu et al. 2011).

\begin{tabular}{lc}
\hline Parameter & Value \\
\hline$h$ & 0.703 \\
$\Omega_{\mathrm{b}}$ & 0.0451 \\
$\Omega_{\mathrm{m}}$ & 0.2711 \\
$\Omega_{\mathrm{DE}}$ & 0.729 \\
$A_{s}$ & $2.42 \times 10^{-9}$ \\
$n_{s}$ & 0.966 \\
\hline
\end{tabular}

Since IDE introduces a scale dependence in the matter growth factor and non-negligible DE perturbations in the Poisson equation (9), the scale-dependent PNG bias for IDE models becomes

$\Delta b(k, z)=3 f_{\mathrm{NL}}\left[b_{\mathrm{G}}(z)-1\right] \frac{\delta_{\text {crit }} \Omega_{m}}{D_{\mathrm{c}}(k, z)[1+\mu(k, z)] T(k)} \frac{H_{0}^{2}}{k^{2}}$,

where the effect of IDE appears in the scale dependence of $D_{\mathrm{c}}$ and in the factor

$\mu=\frac{\dot{\rho}_{\phi}}{\dot{\rho}_{\mathrm{c}}}\left[1-\left(\frac{\dot{\rho}_{\phi}}{\dot{\rho}_{c}}\right)\right]^{-1} \frac{1}{D_{\phi}}$,

where $D_{\phi} \equiv \Delta_{\phi} / \Delta_{\phi}(z=\infty)$ is the DE growth factor and $\mu$ depends on the coupling parameter $\beta$ though the background equations (1) and (2). We can notice that on very large scales, $D_{\mathrm{c}}(k, z)$ behaves as $\sim k^{-2}$.

\section{LINEAR HALO POWER SPECTRUM}

In this section, we will illustrate the degeneracy between IDE and PNG by computing the halo power spectrum on linear scales for both models and for their combination.

The halo power spectrum is given in general by

$P_{\mathrm{h}}(k, z)=\left[b_{\mathrm{G}}(z)+\Delta b(k, z)\right]^{2} P_{\mathrm{m}}(k, z)$.

In order to compute this we first numerically solve equations (10) and (11) for the growth factors $D_{i}$, and then calculate the matter power spectrum $P_{\mathrm{m}}(k, z)$ using (Ade et al. 2016d):

$P_{\mathrm{m}}(k, z)=A_{s}^{2}\left(\frac{k}{k_{p}}\right)^{n_{s}} T^{2}(k)\left[\frac{D_{\mathrm{c}}(k, z)}{D_{\mathrm{c}}(k, 0)}\right]^{2}$,

where $n_{\mathrm{s}}$ is the spectral index, $A_{\mathrm{s}}$ is the spectral amplitude, and $k_{\mathrm{p}}$ is the pivot scale. We use CAMB (Lewis, Challinor \& Lasenby 2000) to compute the transfer function $T(k)$. We then apply the bias relation, equation (18), to the matter power spectrum as given in equation $(20){ }^{2}$ We adopt the cosmological parameters given in Table 1 .

In computing the growth rate of CDM density perturbations we consider both the case where large-scale perturbations in the DE scalar field are properly taken into account $\left(\Delta_{\phi} \neq 0\right)$ and the case where such perturbations are artificially set to zero $\left(\Delta_{\phi}=0\right)$. The latter case, while being not fully consistent, allows us to match the approximations adopted in the numerical treatment that we will discuss below and to obtain a more direct correspondence between the PNG and IDE parameters that are expected to provide a strong degeneracy in the non-linear regime under such approximations. In Fig. 1, we show the ratio of the linear halo power spectrum to the fiducial $\Lambda \mathrm{CDM}$ model at $z=0$ for the cases given in Table 2 .

${ }^{2}$ For the Gaussian bias, we use the ansatz $b_{G}=\sqrt{1+z}$.
These values of $f_{\mathrm{NL}}$ are obtained by minimizing the residual $1-P_{h} / P_{h}^{\Lambda \mathrm{CDM}}$ for the combined model, i.e. they correspond to the values of maximum degeneracy for a $\mathrm{DE}-\mathrm{CDM}$ coupling parameter $\beta=0.05$ for the cases $\Delta_{\phi} \neq 0$ and $\Delta_{\phi}=0$. Clearly, the $k^{-2}$ signal, assuming $\Delta_{\phi}=0$, is larger and therefore the amount of PNG to be degenerate with it is bigger. Therefore, for these combinations of parameters, as clearly seen in Fig. 1, IDE and PNG are strongly degenerate with each other, in the sense that their combination is indistinguishable from the fiducial $\Lambda \mathrm{CDM}$ case. ${ }^{3}$

Although these derived values of $f_{\mathrm{NL}}$ are at least one order of magnitude larger than currently allowed by observational constraints, we will continue to use these values as a toy example of the IDE-PNG degeneracy. Realistic values for $\left|f_{\mathrm{NL}}\right|$ with the standard assumption of a scale-independent amplitude of PNG would have too weak effects on non-linear structure formation to significantly influence the observational features for any non-negligible coupling parameter $\beta$. On the other hand, scale-dependent PNG (see e.g. Liguori et al. 2010; Renaux-Petel 2015), where $f_{\mathrm{NL}}(k)$ evolves with wavenumber $k$, may still provide an effective $f_{\mathrm{NL}}=\mathcal{O}\left(10^{2}\right)$ at scales relevant for non-linear structure formation, while remaining consistent with current bounds around the Planck pivot scale $k=0.05 h^{-1} \mathrm{Mpc}$.

In order to model the mimicking degeneracy relation between $\beta$ and $f_{\mathrm{NL}}$ that is illustrated in Fig. 1, we repeat the procedure of minimizing the residual $1-P_{h} / P_{h}^{\Lambda \mathrm{CDM}}$ for a wide range of the parameters $\beta$ and $f_{\mathrm{NL}}$, for both perturbed and non-perturbed DE cases. We find that relation

$f_{\mathrm{NL}}=\zeta \beta^{-\gamma}$

where $\zeta$ and $\gamma$ are constants, provides a good fit, with exponent $\gamma \approx 1.8$. This is shown in Fig. 2, where the numerical results are overplotted with the fitting function equation (22) for perturbed and non-perturbed DE cases. Note that the degeneracy slope $\gamma$ increases if we assume non-perturbed dark energy.

\section{DEGENERACY ON NON-LINEAR SCALES}

It is well known that IDE and PNG separately imprint characteristic features in the non-linear regime of structure formation, which can be tested through different observational probes. For example, IDE affects the high-mass tail of the halo mass function (HMF) by enhancing the abundance of haloes (Cui et al. 2012), while PNG impacts the number of massive CDM haloes, suppressing (increasing) it for negative (positive) $f_{\mathrm{NL}}$ (see e.g. Grossi et al. 2009; Wagner et al. 2010). It is therefore plausible that some form of degeneracy may appear also at these non-linear scales, and in particular that the combination of IDE with a negative value of $f_{\mathrm{NL}}$ for PNG may result in a HMF hardly distinguishable from the reference $\Lambda \mathrm{CDM}$ case at all masses.

IDE also shows distinctive features on other observational probes, including higher order correlation functions and non-linear bias, in a similar way to PNG (Desjacques, Seljak \& Iliev 2009; Wagner \& Verde 2012; Moresco et al. 2014). IDE further affects the structural properties of CDM haloes and voids (Baldi 2011b, 2014; Pollina et al. 2016, 2017; Giocoli et al. 2013), and PNG is also expected to show significant effects on these probes (Abel, Hahn \& Kaehler 2012; Neyrinck \& Yang 2013; Sutter et al. 2014).

\footnotetext{
${ }^{3}$ We chose $\beta>0$ and $f_{\mathrm{NL}}<0$ because the same degeneracy does not apply for negative $\beta$ in the Newtonian approximation, since the coupling enters also as a $\beta^{2}$ term in equation (14). This means that for $\beta<0$ and $f_{\mathrm{NL}}>0$, we do not expect a degeneracy in the non-linear regime.
} 


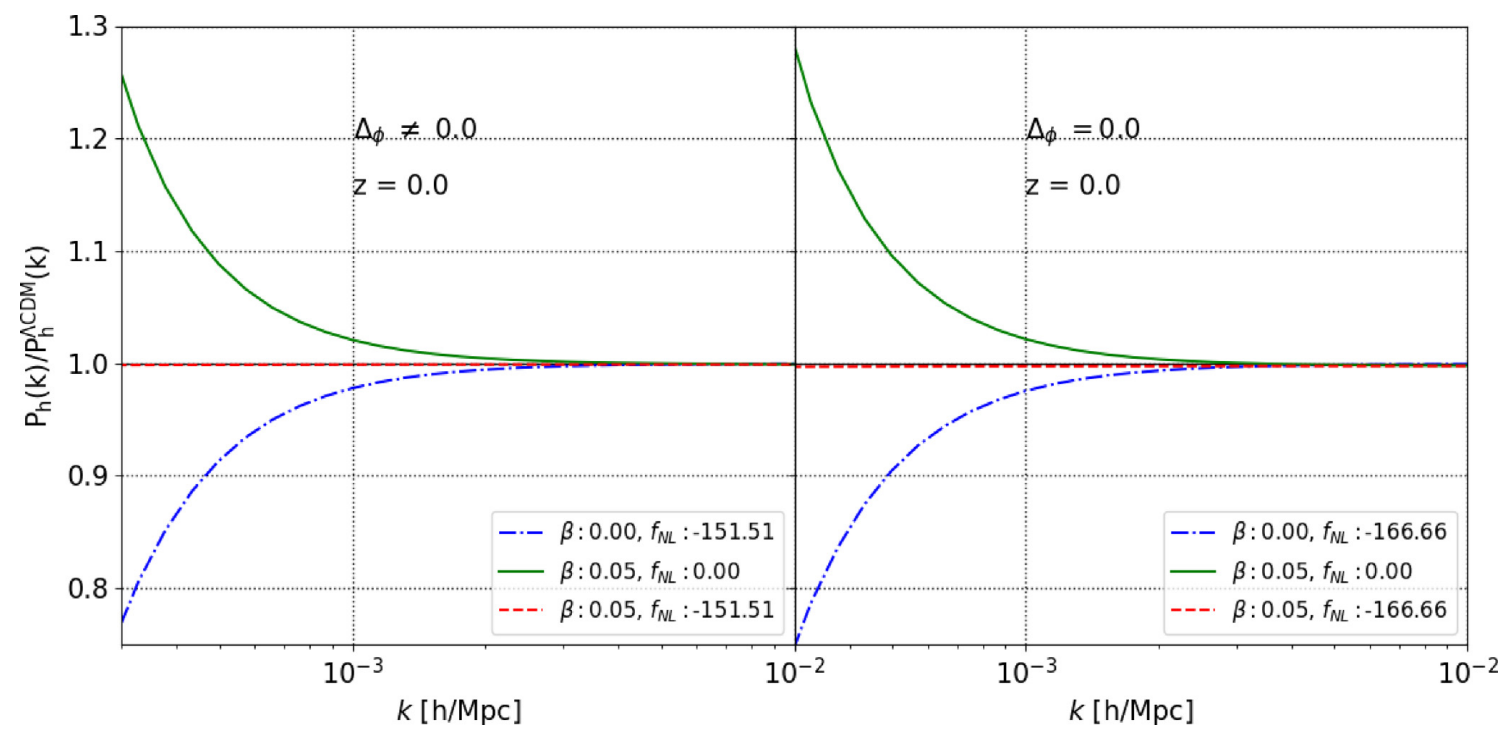

Figure 1. The ratio of the linear halo power spectrum to the standard $\Lambda \mathrm{CDM}$ case for the models (I-VI) given in Table 2 at $z=0$, assuming perturbed DE (left-hand panel) and non-perturbed DE (right-hand panel).

Table 2. Different values of $\beta$ and $f_{\mathrm{NL}}$ parameters used in this paper.

\begin{tabular}{lccc}
\hline & $\beta$ & $f_{\mathrm{NL}}$ & $\mathrm{DE}$ \\
\hline I & 0.05 & 0.0 & $\Delta_{\phi} \neq 0$ \\
II & 0.05 & 0.0 & $\Delta_{\phi}=0$ \\
III & 0.0 & -151.51 & $\Delta_{\phi} \neq 0$ \\
IV & 0.0 & -166.66 & $\Delta_{\phi}=0$ \\
V & 0.05 & -151.51 & $\Delta_{\phi} \neq 0$ \\
VI & 0.05 & -166.66 & $\Delta_{\phi}=0$ \\
\hline
\end{tabular}

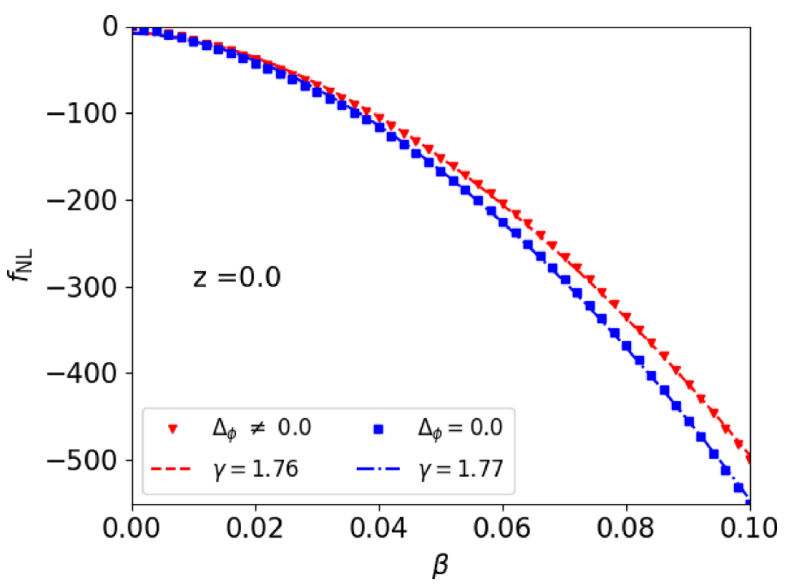

Figure 2. The $\beta-f_{\mathrm{NL}}$ mimicking degeneracy relation at redshift $z=0$, overplotted with the fitting function defined in equation (22), for the fitted values of $\gamma$.

This implies that the mimicking degeneracy which we have found at linear scales for the halo power spectrum may persist (fully or partly) in some small-scale non-linear observables, while it may be broken by others. In the following, we test the linear degeneracy relation, defined in equation (22), on non-linear scales by analysing a suite of cosmological $N$-body simulations that include IDE and PNG, both separately and in a combined way. To this end, we will consider various non-linear probes, starting from the non-linear matter power spectrum and the halo-matter bias to the statistical and structural properties of CDM haloes and voids.

\section{1 $N$-body simulations}

In order to consistently account for the effects of IDE in the nonlinear regime, we made use of a modified version of the parallel TREEPM $N$-body code GADGET (Springel 2005) that incorporates all the specific features of the coupling between DE and CDM, i.e. modified background expansion, CDM particle mass time variation, the extra friction, and the fifth force acting on CDM particles (see Baldi et al. 2010, for a detailed description of the modified $N$-body algorithm). The simulations follow the evolution of $1024^{3} \mathrm{CDM}$ particles within a periodic cosmological box of $1 \mathrm{~h}^{-1} \mathrm{Gpc}$ per side, for all the cosmological parameters given in Table 1, with a mass resolution at $z=0$ of $5.84 \times 10^{10} \mathrm{M}_{\odot} h^{-1}$ and softening length $\epsilon=24.42 h^{-1} \mathrm{kpc}$. Our numerical implementation of IDE assumes that $\mathrm{DE}$ perturbations are negligible in relation to structure formation processes compared to the dominant effects of background evolution, extra friction, and fifth force. This is a valid approximation on sub-horizon scales; it becomes less accurate at scales comparable with the cosmic horizon, but this is beyond the fundamental mode of our $1 h^{-1} \mathrm{Gpc}$ boxes. For this reason, we have chosen to consider the same approximation (i.e. $\Delta_{\phi}=0$ ) to select our combination of values for the parameters $\beta$ and $f_{\mathrm{NL}}$, so as to ensure consistency between the degeneracy relation displayed in Fig. 2 and the outcomes of our $N$-body simulations at small scales.

In order to generate the initial conditions for $N$-body simulation of all models considered in this paper, we slightly modified the publicly available code 2LPTic (Scoccimarro et al. 2012). The algorithm implements non-Gaussian initial conditions with external Hubble and growth functions consistent with IDE modifications.

The non-Gaussian initial conditions are generated for local-type PNG with an extra non-Gaussian term according to equation (15), where $\Phi_{\mathrm{G}}$ is a random realization of a Gaussian field with the primordial power spectrum $P(k) \propto k^{n_{s}-1}$. Then, the linear density field $\delta_{\mathrm{c}}$ is obtained from the non-Gaussian potential $\Phi$ through the 


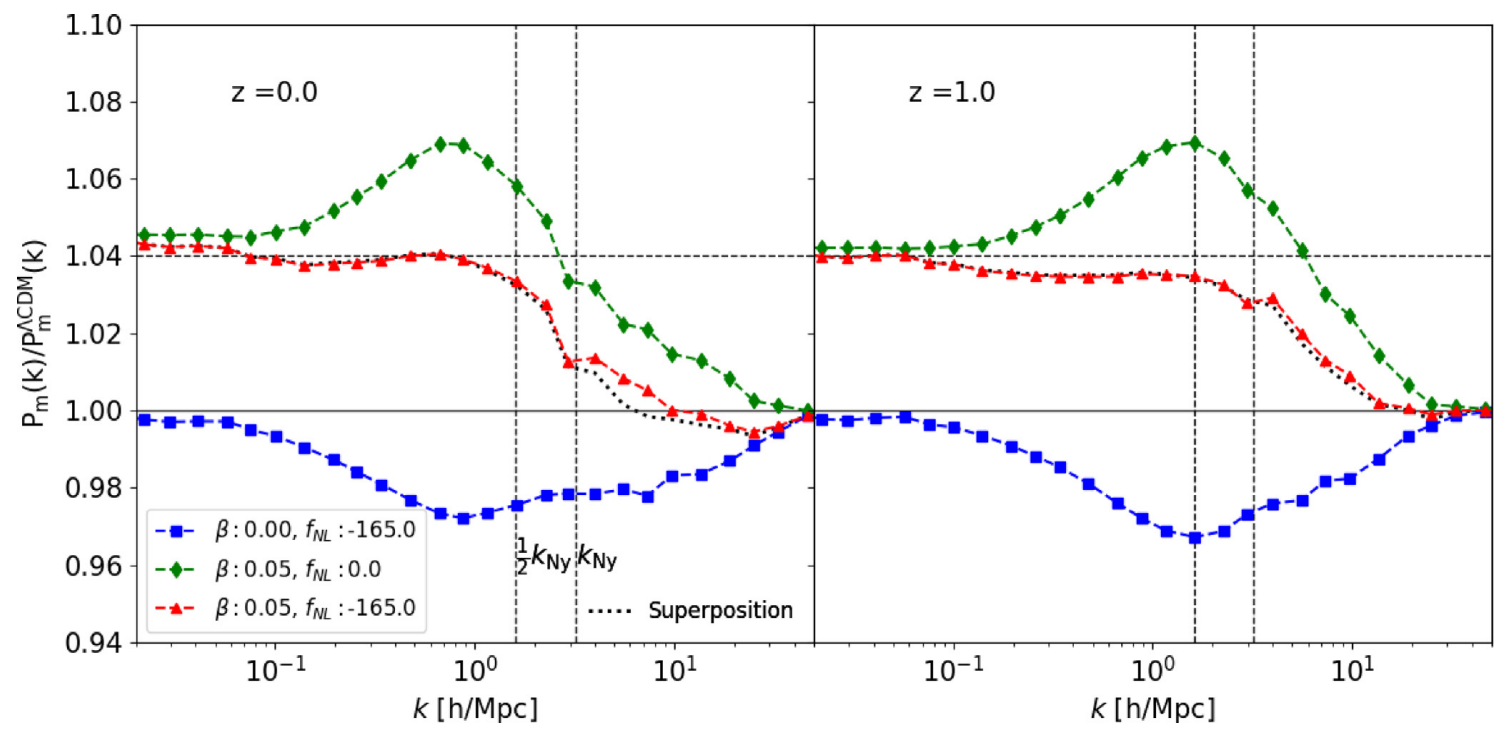

Figure 3. The non-linear matter power spectrum with IDE, PNG, and their combination, relative to the reference $\Lambda$ CDM spectrum, at $z=0$ (left-hand panel) and $z=1$ (right-hand panel). The dotted black curve shows the superposition spectra (IDE-only + PNG-only). The black dashed vertical lines show the Nyqvist frequency and half of it.

Poisson equation:

$\delta_{\mathrm{c}}=\frac{2}{3} \frac{k^{2}}{H_{0}^{2}} \frac{D_{\mathrm{c}}(z)}{\Omega_{\mathrm{c}}} T(k) \Phi$,

where the transfer function $T(k)$ is computed using CAMB (Lewis et al. 2000) for the fiducial $\Lambda \mathrm{CDM}$ cosmology. We assume the transfer function is not affected by the late-time interaction (Baldi et al. 2010; Baldi 2012b). The growth function $D_{\mathrm{c}}$ for all models is normalized at $z_{\mathrm{CMB}} \approx 1100$ to directly compare the impact of IDE on the structure growth in the period between $z_{\mathrm{CMB}}$ and the present time. For PNG, we set $f_{\mathrm{NL}}=-165.0$ as the value corresponding to the interaction rate $\beta=0.05$ on linear scales (with $\Delta_{\phi}=0$ ), as determined by equation (22).

Particle positions are then displaced from a homogeneous glass distribution (Baugh, Gaztanaga \& Efstathiou 1995) using the Zel'dovich approximation (Zeldovich 1970) according to the displacement field $\delta_{\mathrm{c}}$ at the initial redshift $z_{i}=49$. In order to compute particle initial velocities, we used the relation $v(k, z) \propto f(z) \delta(k, z)$, where the growth rate function $f(z) \equiv-d \ln D_{\mathrm{c}} / d \ln (1+z)$ is derived for each model by solving equations (14) for the growth function. For the IDE-PNG combined model, we apply the growth function of IDE after transforming the initial Gaussian potential to the nonGaussian form according to equation (15). In order to minimize the sampling variance, we used the same initial random seed for all the simulations.

\section{RESULTS}

In this section, we present the main results of our numerical simulations of IDE, PNG, and the combined IDE-PNG extensions rescaled with respect to the fiducial $\Lambda \mathrm{CDM}$ model. We focus mainly on the non-linear matter power spectrum, the halo-matter bias and the statistical and structural properties of CDM haloes and voids.

\subsection{The non-linear matter power spectrum}

We computed the non-linear matter power spectrum for each simulation by calculating the density field using a Cloud-in-Cell mass assignment on a cubic grid with the same resolution as the Particle Mesh grid used for the integration of the $N$-body system (i.e. $1024^{3}$ ). According to this procedure, the non-linear matter power spectrum is determined up to the Nyquist scale, $k_{\mathrm{Ny}}=\pi N / L \sim 3.2 \mathrm{~h} \mathrm{Mpc}^{-1}$. We truncate the resulting power spectrum at the $k$-mode where the shot noise is below 20 per cent of the measured power. From the simulated power spectra, we can estimate the effects of IDE, PNG and, for the first time, the joint effects of IDE and PNG, on linear and non-linear scales at different redshifts.

In Fig. 3, we display the ratio of the non-linear matter power spectrum for IDE, PNG and their combination, to that of the standard cosmological model, at $z=0$ and $z=1$. The plots show the following features.

IDE with Gaussian initial conditions (dashed green curve with solid diamonds) - shows the expected scale-dependent power enhancement at non-linear ranges due to the combined effects of the fifth force and of the extra friction associated with the DE$\mathrm{CDM}$ interaction. Also, since we normalize the power spectrum at the redshift of the $\mathrm{CMB}$, the normalization at linear scales $\left(\sigma_{8}^{\Lambda \mathrm{CDM}}=0.809\right)$ is increased by about 5 per cent relative to the standard model (i.e. $\sigma_{8}^{\mathrm{IDE}}=0.825$ ), due to the higher linear growth rate in the IDE case (Baldi 2011a); this is consistent with previous works (see e.g. Baldi 2011b). At higher redshift (right panel) the non-linear power spectrum enhancement due to the IDE fifth force is slightly reduced, with the peak ratio shifted towards smaller scales.

Non-Gaussian initial conditions in $\Lambda \mathrm{CDM}$ (dashed blue curve with solid squares) - shows the expected suppression of power at small scales, relative to the standard $\Lambda \mathrm{CDM}$ case. The deviation is larger at higher redshifts and the minimum shifts towards smaller scales, in agreement with predictions from the halo model presented in Fedeli \& Moscardini (2010).

IDE and PNG combined (dashed red curve with solid triangles) - the ratio no longer shows any significant scale dependence down to ranges corresponding to the location of the peak/minimum in the ratio for the two separate models. The difference in the power normalization at linear scales associated with the enhanced growth rate in IDE remains unchanged. This seems to indicate a mimicking 


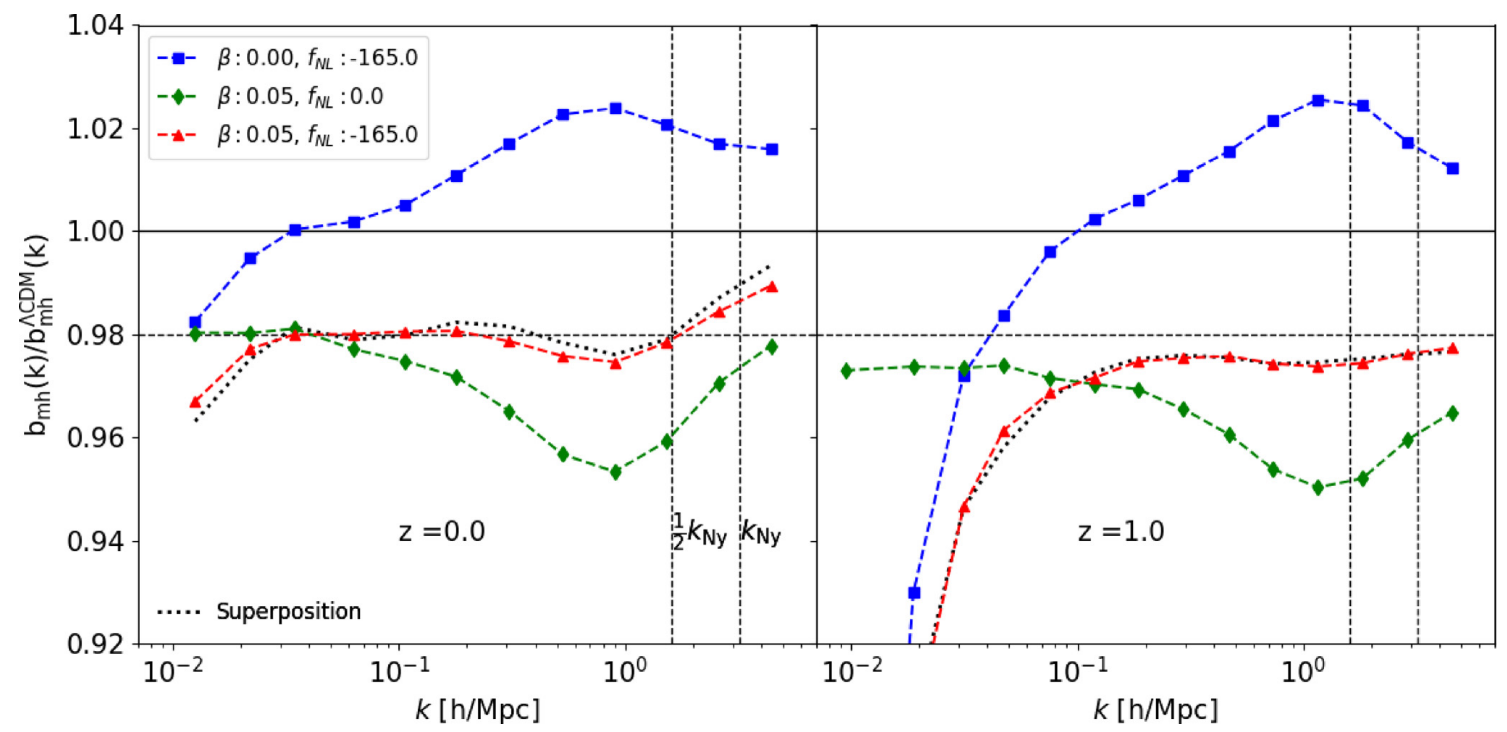

Figure 4. As in Fig. 3, for the halo-matter bias. Clearly, IDE shows no sign of scale-dependence on large scales.

degeneracy between IDE and PNG in the matter power spectrum on non-linear scales while there is no degeneracy on linear scales. Remarkably, the figure shows that there is a non-linear mimicking degeneracy for the same combination of parameters that produce mimicking degeneracy in the halo power spectrum at much larger scales, as described by equation (22) and Fig. 2.

In the figures we have also overplotted, for comparison, a black dotted curve representing a simple superposition of the two effects, i.e. the PNG-only (blue squares) deviation times the IDEonly (green diamonds) deviation. The very good agreement of this simple prediction with the actual power measured from the combined IDE-PNG simulation seems to indicate that the two effects acting on structure formation are decoupled - which suggests that full combined $N$-body simulations may be unnecessary in order to compute the combined power spectrum for other combinations of $\beta$ and $f_{\mathrm{NL}}$.

\subsection{Halo-matter bias}

Following the standard hierarchical clustering scenario of structure formation, haloes and galaxies are biased tracers of the underlying matter distribution. In this section, we compute the linear bias between haloes and the underlying dark matter density field, as the ratio between the halo-CDM cross power spectrum and the auto power spectrum in Fourier space:

$b_{\mathrm{hm}}(k)=\frac{P_{\mathrm{hm}}(k)}{P_{\mathrm{mm}}(k)}$.

(We suppress the $z$-dependence for simplicity.) This bias estimator is used to avoid shot-noise (Smith, Scoccimarro \& Sheth 2007; Baldauf et al. 2010, 2013; Hamaus et al. 2010), and we follow the approach of Villaescusa-Navarro et al. (2014) for the computation of the two power spectra.

In Fig. 4, we show the ratio of the halo-matter bias for the IDE, PNG, and IDE-PNG models, relative to the fiducial $\Lambda$ CDM model. As expected, PNG introduces a clear scale-dependence at large scales. On the contrary, the bias in the IDE model appears to have a slightly lower normalization than $\Lambda \mathrm{CDM}$ though retaining the same evolution with scale as the standard scenario. This different behaviour is most visible at higher redshifts, as shown in the right-hand panel of Fig. 4, where the scale-dependence of the PNG simulation is stronger. On non-linear scales, both PNG and IDE show a maximum deviation relative to the reference model but in opposite directions, with the amplitudes of the peak/minimum increasing and their position moving towards smaller scales at higher redshifts. These outcomes are all consistent with the previous literature (Matarrese \& Verde 2008; Desjacques et al. 2009; Marulli, Baldi \& Moscardini 2012; Moresco et al. 2014) and qualitatively show how the halo bias is affected at similar scales for both IDE and PNG.

For the combined IDE-PNG scenario, we find that at $z=0$ the halo bias retains some scale-dependence on large scales, i.e $k<0.05 h \mathrm{Mpc}^{-1}$, while it is nearly scale-independent on scales $0.05 h \mathrm{Mpc}^{-1}<k<0.5 h \mathrm{Mpc}^{-1}$. Furthermore, it retains the lower normalization that characterizes the IDE model at all scales. This combination of the two effects is more clear at higher redshift, where we can clearly identify two distinct regions for scale-dependent $(k<$ $\left.0.1 h \mathrm{Mpc}^{-1}\right)$ and scale-independent $\left(k>0.1 h \mathrm{Mpc}^{-1}\right)$ deviations from the reference model. Also in this case, the simple superposition of the two separate effects, very accurately reproduces the behaviour of the combined IDE-PNG simulation, thereby suggesting that the two phenomena act on the biasing of collapsed structures independently.

Similar to the non-linear matter power spectrum, the halo-matter bias satisfies the $\beta-f_{\mathrm{NL}}$ degeneracy relation, equation (22), on nonlinear scales, while this is broken at larger scales. We argue that this may be due to the fact that our $N$-body implementation of IDE as discussed above (see also Baldi 2011b), does not account for the scale-dependent growth function on large scales due to CDMDE coupling and the contribution of large-scale DE perturbations (i.e. it assumes the approximations $D_{\mathrm{c}}(k, z) \simeq D_{\mathrm{c}}(z)$ and $\Delta_{\phi}=0$ ). Therefore, including the effects of large-scale CDM-DE coupling and DE perturbations should boost in a scale-dependent way the IDE linear power spectrum and consequently the halo-matter bias on large scales. This would recover the result of a mimicking degeneracy at all scales that was obtained from linear perturbation theory (Sec. 3). A proper verification of this conjecture would require major modifications to our $N$-body codes, that go beyond the scope of the present paper, and we defer an extensive study on this subject to future works. 


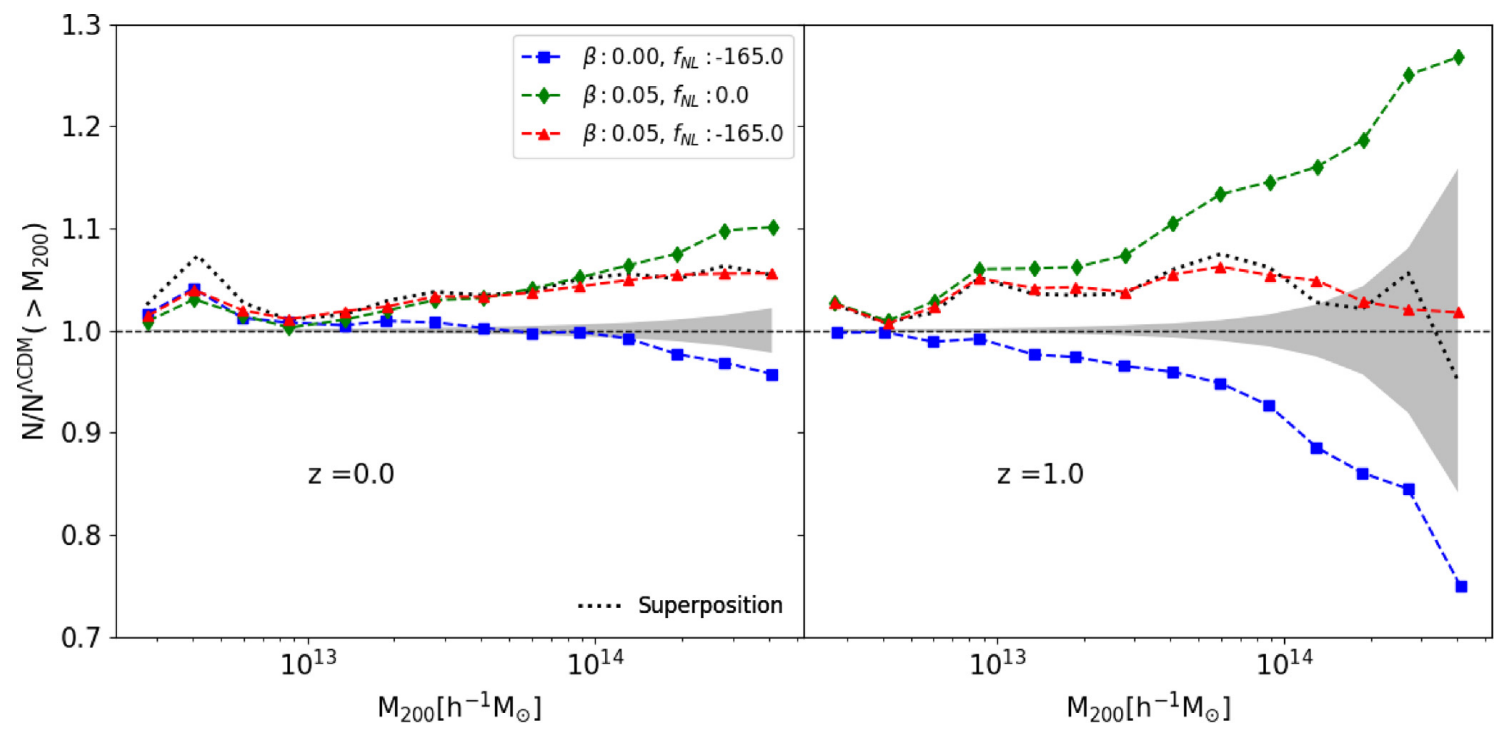

Figure 5. As in Fig. 3, for the halo mass function. The grey region represents the propagated Poissonian error of the number counts of haloes in each bin.

\subsection{Statistical and structural properties of CDM haloes}

In this section, we test the $\beta-f_{\mathrm{NL}}$ degeneracy relation in the statistical and structural properties of CDM haloes.

\subsubsection{The halo mass function}

We identified collapsed haloes in our simulations following a standard procedure, amounting to a first identification of particle groups by means of a Friends-of-Friends (FoF) algorithm with linking length $l=0.2 \bar{d}$, where $\bar{d}$ indicates the mean inter-particle separation. On top of these FoF haloes we run the SUBFIND algorithm (Springel et al. 2001) in order to identify gravitationally bound substructures present within each group. The latter procedure allows to assign to each FoF group the virial mass $\mathbf{M}_{200}$ of its primary substructures, defined as the mass of a spherical region with its centre on the particle with the halo's minimum potential enclosing a mean overdensity equal to 200 times the critical density of the universe.

Given these halo catalogues, we computed the halo mass function for IDE, PNG, and the combined IDE-PNG models by binning the halo masses into 13 logarithmically equally spaced mass bins over the mass range $2.0 \times 10^{12} \mathrm{M}_{\odot} \mathrm{h}^{-1}-5.0 \times 10^{14} \mathrm{M}_{\odot} \mathrm{h}^{-1}$. The lower mass bound is set by the minimum halo mass resolved in the fiducial $\Lambda \mathrm{CDM}$ model, composed of at least 20 particles.

In Fig. 5, we show the ratio of the cumulative HMF to the $\Lambda$ CDM model for IDE, PNG, and the combined IDE-PNG models. As expected, IDE enhances the abundance of large mass haloes with respect to the standard $\triangle \mathrm{CDM}$ case, while PNG shows on the contrary a suppression of the abundance of haloes in the high-mass tail, consistent with previous results (Wagner et al. 2010; Cui et al. 2012)

The combined IDE-PNG model shows some level of degeneracy with the standard $\Lambda \mathrm{CDM}$ cosmology at $z=0$, with the combined mass function being slightly lower than in the pure IDE case. The degeneracy becomes more clear, given the larger amplitude of the individual effects, at higher redshifts $(z=1)$, where the IDE and PNG deviations from the reference model reach about $25-30$ per cent at the largest masses with abundance suppression by only 5 per cent in the combined case. Furthermore, the exponential dependence on halo mass of the deviation with respect to $\Lambda \mathrm{CDM}$ is also sig-

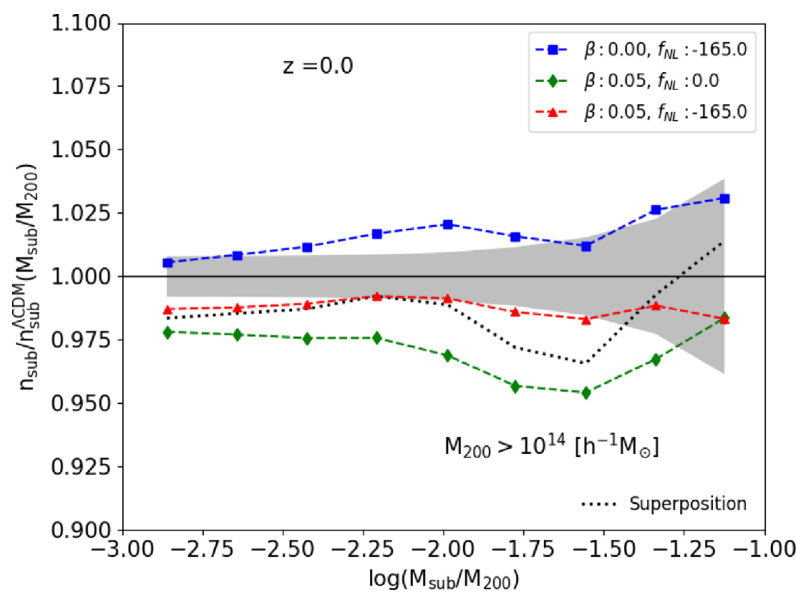

Figure 6. The subhalo mass function for the cosmologies under investigation at $z=0$. The grey region represents the propagated Poissonian error of the number counts of subhaloes in each bin and the dotted black line represents the superposition of IDE and PNG models.

nificantly weakened in the combined model. None the less, as a mimicking degeneracy is never fully attained, the halo mass function seems not to follow the degeneracy relation of equation (22), thereby providing a possible way to disentangle these phenomena.

The simple superposition of IDE and PNG models reasonably agrees with the combined IDE-PNG simulation except in the low mass end of the halo mass function at $z=0$ (see left-hand panel of Fig. 5) where some disagreement appears. This presumably could be related to the poor resolution of small mass haloes thereby arising due to numerical artefacts associated with the specific halo finder that we employed.

\subsubsection{The subhalo mass function}

As a further statistic of structure properties at small scales, we computed - for all our simulated cosmologies - the subhalo mass function, defined as the number of subhaloes of mass $\mathbf{M}_{\text {sub }}$ within a main halo of virial mass $\mathbf{M}_{200}$. In Fig. 6, we display the ratio of 


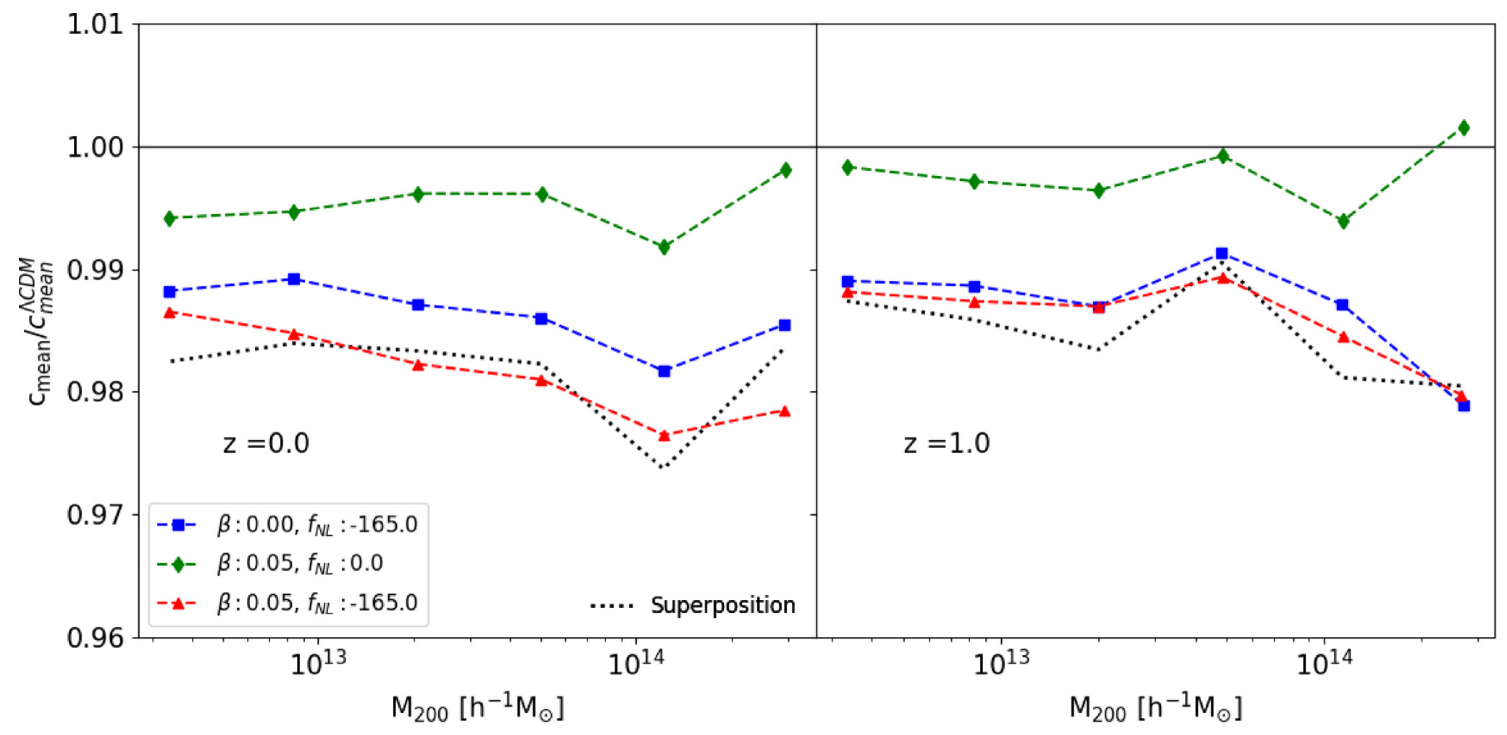

Figure 7. As in Fig. 3, for the concentration-mass relation.

the subhalo mass function with respect to the measurements in the $\Lambda \mathrm{CDM}$ simulation, as a function of the mass ratio $\mathrm{M}_{\text {sub }} / \mathrm{M}_{200}$. In order to avoid resolution effects, we consider only subhaloes hosted by cluster-size haloes, i.e. systems with $\mathrm{M}_{200}>10^{14} \mathrm{M}_{\odot} \mathrm{h}^{-1}$. We underline to the reader that the measured subhalo counts in the $\Lambda \mathrm{CDM}$ model are characterized by the typical slope of approximately -1 consistent with different previous findings (Gao et al. 2004; Giocoli et al. 2010; Despali \& Vegetti 2017).

As can be seen from the figure, IDE suppresses the abundance of sub-structures over the whole range of subhalo fractional mass, even though the effect is small (about 3-5 per cent). On the contrary, PNG enhances the abundance of subhaloes up to about 4 per cent (for the highest values of the subhalo fractional mass) over the same mass range. The combined IDE-PNG case shows again a quite clear degeneracy, with a suppression never exceeding $\approx 1$ per cent, marginally consistent with the Poissonian error range of the $\Lambda \mathrm{CDM}$ model. The simple superposition of IDE and PNG models is in reasonable agreement with the combined IDE-PNG simulation. These results underline that the $\beta-f_{\mathrm{NL}}$ degeneracy relation seems to remain valid also at the level of CDM halo sub-structures.

\subsubsection{Halo concentration}

Finally, we conclude our investigation of the combined effects of IDE and PNG on structural properties of collapsed haloes by computing the average halo concentration as a function of halo mass, which is usually known as the concentration-mass relation (Zhao et al. 2009; Giocoli, Tormen \& Sheth 2012). In order to compute the concentrations for the haloes identified in our simulations, we adopt the NFW formula used in Springel et al. (2008):

$\delta_{\mathrm{con}}=\frac{200}{3} \frac{c^{3}}{\ln (1+c)-c /(1+c)}=14.426\left(\frac{V_{\max }}{H_{0} r_{\max }}\right)^{2}$,

where $\delta_{\text {con }}$ is the characteristic overdensity, $c$ is the halo concentration, $V_{\max }$ is the maximum circular velocity of the halo attained at radius $r_{\max }$. In Fig. 7, the ratio of the concentration-mass relation of IDE, PNG, and the combined IDE-PNG models relative to $\Lambda \mathrm{CDM}$ is presented at $z=0$ (left-hand panel) and $z=1$ (right-hand panel).
As expected, IDE haloes are found to be less concentrated with respect to the fiducial $\Lambda \mathrm{CDM}$ case, in agreement with results given in Baldi (2011b). Similarly, PNG with $f_{\mathrm{NL}}<0$ also suppresses halo concentrations (the opposite would occur for a positive $f_{\mathrm{NL}}$ ). Therefore, for the first time we encounter an observational probe showing deviations from $\Lambda \mathrm{CDM}$ pointing in the same direction for IDE and our negative $f_{\mathrm{NL}}$ PNG scenarios.

The combined IDE-PNG simulation, accordingly, shows an even stronger suppression of the concentration-mass relation relative to the $\Lambda \mathrm{CDM}$ model than the two individual models separately. The effects are less pronounced at higher redshifts, while the trends and the relative ordering of the various models is preserved. Superposition of the individual effects of IDE and PNG seems to agree well with the combined simulation. This however indicates that the $\beta-f_{\mathrm{NL}}$ degeneracy is broken for the CDM halo concentration-mass relation, which might then represent another direct way to disentangle the models, when combined with another more degenerate probe. It is also reasonable to emphasize that this effect is relatively small; only future wide field observational campaigns - like the future ESA-mission Euclid (Laureijs et al. 2011) - will be able to collect the large number of galaxy groups and clusters (Sartoris et al. 2016) necessary for these tests.

\subsection{Statistical and structural properties of cosmic voids}

In this section, we move our focus to underdense regions of the universe by testing whether cosmic voids also follow the $\beta-f_{\mathrm{NL}}$ degeneracy relation. In order to identify cosmic voids in our set of simulations, we employ the publicly available void finder VIDE (Sutter et al. 2015), which is based on the ZOBOV algorithm (Neyrinck 2008). The cosmic void identification is mainly done by means of a Voronoi tessellation scheme that associates a polyhedrical cell to each particle tracing the CDM density field. Subsequently, cell volumes are compared in order to identify local density minima, i.e. cells with a larger Voronoi volume than all their surrounding cells. A hierarchy of identified voids is then obtained via the watershed transform algorithm (Platen, van de Weygaert \& Jones 2007), by joining Voronoi cells around a local density minimum. In our analysis, we consider only voids with a central density that is below the density of the universe by 20 per cent and a lower density con- 


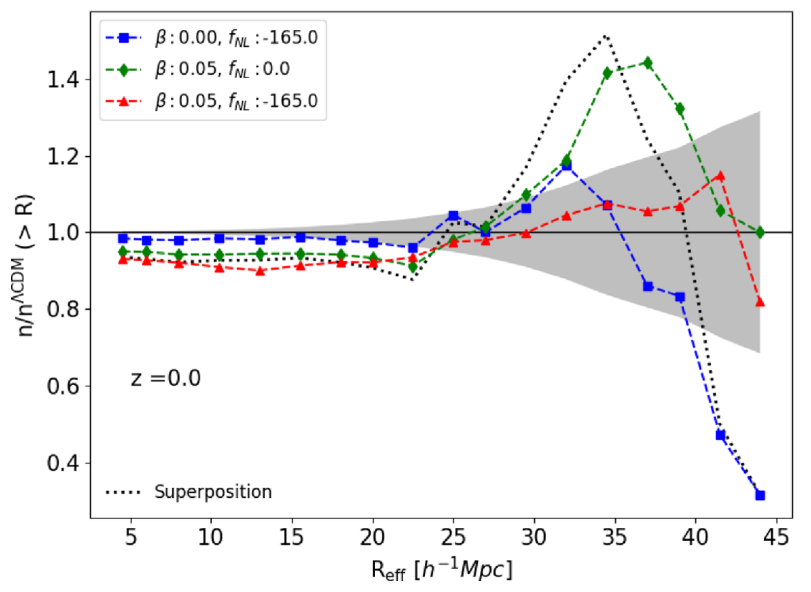

Figure 8. As in Fig. 6, for the void number function.

trast limit 1.57, corresponding to a probability of voids arising from Poisson noise below $\sim 5$ per cent, i.e only voids at $2 \sigma$ confidence level are considered (Neyrinck 2008).

\subsubsection{Void number function}

As a first statistics for cosmic voids, we study their abundance as a function of the void effective radius $R_{\text {eff }}$, defined as the radius of a sphere centred on the most underdense particle of a void and having the same volume as the Voronoi volume of the void:

$V_{\text {void }} \equiv \sum_{i=1}^{N} V_{i}^{p}=\frac{4}{3} \pi R_{\text {eff }}^{3}$.

In Fig. 8 we show the ratio of the void number functions relative to the ones in the $\Lambda \mathrm{CDM}$ cosmology for all models under consideration, as a function of the effective radius $R_{\text {eff }}$ at $z=0$. From the figure we see that IDE suppresses the number of cosmic voids with effective radius $R_{\text {eff }}<25 h^{-1} \mathrm{Mpc}$ by about 5 per cent relative to the $\Lambda \mathrm{CDM}$ case, and correspondingly enhances the abundance of larger voids by up to 40 per cent. The trend is qualitatively similar, though quantitatively weaker (up to ranges $R_{\text {eff }} \approx 30 \mathrm{Mpc} \mathrm{h}^{-1}$ ), for PNG, in agreement with previous results of Kamionkowski, Verde \& Jimenez (2009). However, PNG strongly suppresses void number function at $R_{\mathrm{eff}} \approx 40 \mathrm{Mpc} \mathrm{h}^{-1}$, while IDE enhances it by 40 per cent at the same scales.

The combined IDE-PNG simulation shows suppression of the void number function for radii $R_{\text {eff }}<25 h^{-1} \mathrm{Mpc}$, similar to the IDE case and barely enhances the void abundance at $R_{\text {eff }}>25 h^{-1} \mathrm{Mpc}$ relative to the $\Lambda \mathrm{CDM}$ case, so that it is indistinguishable within the $\Lambda \mathrm{CDM}$ Poisson error range at these radii. As we did for all previous observables, we also compute the simple superposition of the two effects, by taking the product of the two separate deviations with respect to the reference case. For the first time in our analysis, we see that such a superposition fails to reproduce the results of the combined simulation at large void effective radii: this follows from comparing the black dotted curve, representing the analytical superposition, with the blue squares, showing the combined simulation in Fig. 8. In this case we notice that the simple superposition of the two fields tends to be mainly dominated by the IDE not leaving much contribution to the PNG. This suggest that in the full simulation, in void regions, a cross-talk term between the two non-standard extensions emerges moving down the void number counts with respect to the simple superposition.
This suggests that the two phenomena interplay in some way in shaping the growth of large cosmic voids, and cannot be considered as fully independent in this regime. In any case, we notice that the $\beta-f_{\mathrm{NL}}$ degeneracy is fulfilled by the abundance of cosmic voids with large effective radii ( $R_{\text {eff }}>25 h^{-1} \mathrm{Mpc}$ ), while it does not seem to apply at smaller void radii.

\subsubsection{Void density profiles}

To further check the $\beta-f_{\mathrm{NL}}$ degeneracy on cosmic void structural properties, we computed the average void density profiles for two different bins of void radius, namely $0<R_{\text {eff }}<20 h^{-1} \mathrm{Mpc}$ and $20<R_{\text {eff }}<40 h^{-1} \mathrm{Mpc}$. We do this by stacking individual density profiles of 100 randomly selected voids, for each radius bin, corresponding among the different cosmological simulations. We display the ratio of the resulting void mean density profiles in Fig. 9 for all considered models, relative to $\Lambda \mathrm{CDM}$ at $z=0$. The grey area represents the $2 \sigma$ confidence limit, computed by means of a bootstrap re-sampling technique.

Again, we compare the observational signature of the individual IDE and PNG models with their combination. As can be seen from the plot, cosmic voids in the IDE case tend to have a lower inner density than their $\Lambda \mathrm{CDM}$ counterparts. This indicates that cosmic voids are emptier in the IDE case, fully consistent with previous results (see e.g. Pollina et al. 2016). Correspondingly, the compensating overdensity around the effective radius $R_{\text {eff }}$ is found to be more prominent than in $\Lambda \mathrm{CDM}$. On the other hand, PNG shows a negligible effect on cosmic void density profiles. It is then not surprising that the combined IDE-PNG model also shows lower density profiles in the central regions of the voids. This result also shows that cosmic voids do not seem to follow the same degeneracy relation that applies for most of the observables related to properties of the overdense regions of the universe.

\section{DISCUSSION AND CONCLUSIONS}

The concept of observational degeneracy in cosmology arises in several different forms: (1) Parameter Degeneracy represents the existence of large error correlations between different model parameters for specific measurements (Efstathiou \& Bond 1999; Crooks et al. 2003; Tereno et al. 2005; Howlett et al. 2012); (2) Dark Degeneracy reflects the fact that gravitational experiments measure the energy-momentum tensor of the total dark sector and splitting into dark energy and dark matter is arbitrary (Kunz 2009; Aviles \& Cervantes-Cota 2011); (3) Mimicking Degeneracy occurs when cosmological models different from the standard $\Lambda \mathrm{CDM}$ mimic some of its specific features, like background expansion and the growth of matter perturbations (Fay, Nesseris \& Perivolaropoulos 2007; Setare \& Mohammadipour 2013; Fay 2016).

Cosmic degeneracy of IDE has been investigated in the literature (Clemson et al. 2012; Väliviita \& Palmgren 2015), including the partial mimicking degeneracy of IDE and MG (Wei \& Zhang 2008; Koyama et al. 2009; Wei et al. 2013). A mimicking degeneracy between PNG in the power spectrum in the Newtionian approximation, and the correct general relativistic power spectrum with Gaussian initial conditions, has been shown by Bruni et al. (2012) and Jeong, Schmidt \& Hirata (2012). Also, parameter degeneracy has been investigated in the non-Gaussian halo bias by Carbone, Mena \& Verde (2010). Moreover, Abramo \& Bertacca (2017) investigated the degeneracy of large-scale velocity effects on galaxy clustering with the (local) non-Gaussianity parameter $f_{\mathrm{NL}}$, by simulating 


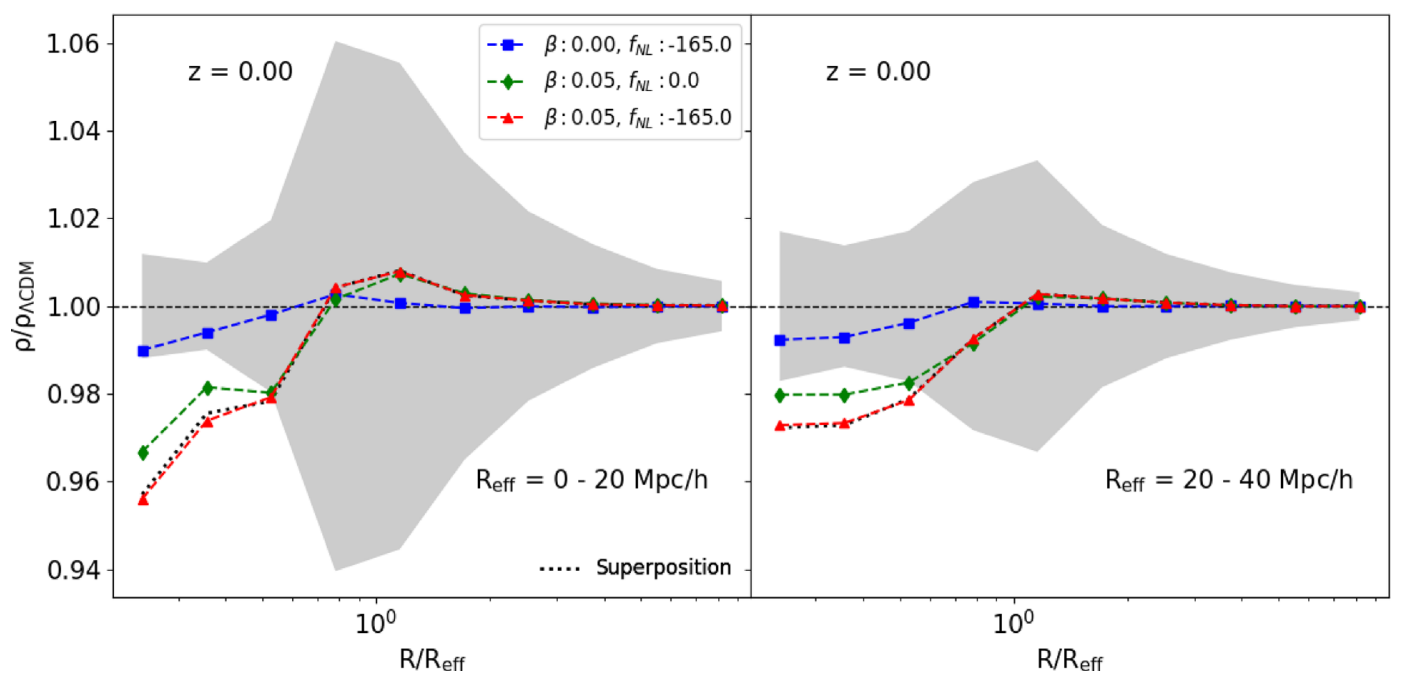

Figure 9. The ratio of the stacked void density profiles in two different ranges of effective radius $R_{\text {eff }}=0-20$ (left-hane panel) and $R_{\text {eff }}=20-40$ (right-hand panel) to the standard model at $z=0$. The grey region represents the $2 \sigma$ bootstrap standard deviation of 1000 re-sampled profiles.

galaxy surveys and combining the clustering of different types of tracers of LSS. They studied how large-scale velocity contributions could be mistaken for the signatures of primordial non-Gaussianity (see also Raccanelli et al. 2014, 2018).

In this paper - as part of a Cosmic Degeneracies paper series (Baldi et al. 2014; Baldi \& Villaescusa-Navarro 2018) - we have considered the mimicking degeneracy between IDE and PNG that was first shown in linear perturbation theory by Hashim et al. (2014). Since IDE can mimic PNG, the possibility exists that we can choose IDE and PNG parameters such that the two effects cancel, i.e. produce standard $\Lambda \mathrm{CDM}$ behaviour. We confirmed this mimicking degeneracy in the halo power spectrum on very large scales, i.e. $k$ $\lesssim k_{\text {eq }}$, based on purely analytical calculations in the linear regime. We then fitted the degeneracy relation with a power law, $f_{\mathrm{NL}} \propto \beta^{-\gamma}$ (depicted in Fig. 2), by minimizing the residual of the halo power spectrum for the combined IDE-PNG model with respect to the mimicked $\Lambda$ CDM model.

To further investigate and validate the $\beta-f_{\mathrm{NL}}$ degeneracy, equation (22), at non-linear scales, we employed a suite of specifically designed $N$-body simulations including the effects of IDE and PNG, both separately and combined with each other. In order to increase the effects under investigation and more easily detect their signatures we chose very large values of the PNG parameter $f_{\mathrm{NL}}$, which are already ruled out by the most recent CMB observations. Still, such values could be achieved at the scales tested by our simulations for simple extensions of the PNG model such as e.g. a scaledependent $f_{\mathrm{NL}}$. We extracted from our simulations a set of standard statistics, and we studied their deviations from the reference Gaussian $\Lambda \mathrm{CDM}$ model. In particular, we did investigate:

- The non-linear matter power spectrum, for which we observed that the mimicking degeneracy persists, remarkably, on non-linear scales in the sense that the scale-dependent deviation with respect the reference $\Lambda \mathrm{CDM}$ scenario characterizing the two separate models at non-linear scales disappears in the combined simulation even though the difference in the linear power normalization due to the enhanced growth rate in IDE is not removed;

- The halo matter bias, for which we find similarly to the nonlinear power spectrum, that the scale-dependence imprinted by the two different models at non-linear scales is also strongly suppressed in the combined simulation while on linear scales such scale-dependent feature is retained and so breaks the observed degeneracy;

- The halo mass function, which also shows some level of degeneracy though not satisfying equation (22) for the degenerate $\beta-f_{\mathrm{NL}}$ values thus allowing us to disentangle the observed degeneracy;

- The subhalo mass function, also showing mimicking degeneracy over the whole subhalo mass range availabe in our simulations;

- The halo concentration-mass relation, which we found to be the first observable to explicitly break the degeneracy as both PNG and IDE have qualitatively the same impact on halo concentrations, namely to suppress concentrations at a given mass with respect to the reference $\Lambda \mathrm{CDM}$ scenario;

- The void number function showing mimicking degeneracy for large voids $\left(R_{\text {eff }}>25-30 h^{-1} \mathrm{Mpc}\right)$ while the degeneracy is broken for smaller void radii;

- and the void density profiles for which, similarly to the case of the concentration-mass relation, the mimicking degeneracy is also not observed at all as both individual models predict a lower inner density of cosmic voids compared to $\Lambda \mathrm{CDM}$.

Therefore, we conclude that measurements of CDM halo and cosmic void internal structural properties, namely halo concentrationmass relation and void density profile would allow us basically to break the degeneracy when combined to any of the other probes that we investigated in this work.

In principle, this degeneracy creates difficulties in identifying the simultaneous presence of IDE and PNG, and in accurately constraining them separately. However, in practice, the degeneracy only arises for values of $\left|f_{\mathrm{NL}}\right|$ that are ruled out by current constraints. Nevertheless, our investigation has shown which non-linear probes could be most useful for improving constraints on IDE and PNG.

\section{ACKNOWLEDGEMENTS}

$\mathrm{MH}, \mathrm{CG}$, and MB acknowledge support from the Italian Ministry for Education, University and Research (MIUR) through the SIR individual grant SIMCODE, project number RBSI14P4IH. CG acknowledges support from the Italian Ministry of Foreign Affairs and International Cooperation, Directorate General for Country Promotion. The simulations described in this work were done on the Sciama High Performance Compute (HPC) cluster which is sup- 
ported by the ICG, SEPNet, and the University of Portsmouth. DB acknowledges partial financial support by ASI grant no. 2016-24H.0. During the preparation of this work DB was also supported by the Deutsche Forschungsgemeinschaft through the Transregio 33, The Dark Universe and Unidad de Excelencia 'María de Maeztu'. RM is supported by the South African SKA Project and the National Research Foundation of South Africa (grant no. 75415), and by the UK Science \& Technology Facilities Council (grant no. ST/N000668/1).

\section{REFERENCES}

Abel T., Hahn O., Kaehler R., 2012, MNRAS, 427, 61

Abramo L. R., Bertacca D., 2017, Phys. Rev. D, 96, 123535

Ade P. A. R. et al., 2016a, A\&A, 594, A13

Ade P. A. R. et al., 2016b, A\&A, 594, A14

Ade P. A. R. et al., 2016c, A\&A, 594, A17

Ade P. A. R. et al., 2016d, A\&A, 594, A20

Ade P. A. R. et al., 2016e, A\&A, 594, A24

Alonso D., Ferreira P. G., 2015, Phys. Rev. D, 92, 063525

Amendola L., 2000, Phys. Rev. D, 62, 043511

Amendola L., 2004, Phys. Rev. D, 69, 103524

Amendola L., Baldi M., Wetterich C., 2008, Phys. Rev. D, 78, 023015

Aviles A., Cervantes-Cota J. L., 2011, Phys. Rev. D, 84, 083515

Baldauf T., Smith R. E., Seljak U., Mandelbaum R., 2010, Phys. Rev. D, 81, 063531

Baldauf T., Seljak U., Smith R. E., Hamaus N., Desjacques V., 2013, Phys. Rev. D, 88, 083507

Baldi M., 2011a, MNRAS, 411, 1077

Baldi M., 2011b, MNRAS, 414, 116

Baldi M., 2012a, MNRAS, 420, 430

Baldi M., 2012b, MNRAS, 422, 1028

Baldi M., 2012c, Annalen Phys., 524, 602

Baldi M., 2014, Phys. Dark Univ., 3, 4

Baldi M., Pettorino V., 2011, MNRAS, 412, L1

Baldi M., Villaescusa-Navarro F., 2018, MNRAS, 473, 3226

Baldi M., Pettorino V., Robbers G., Springel V., 2010, MNRAS, 403, 1684

Baldi M., Villaescusa-Navarro F., Viel M., Puchwein E., Springel V., Moscardini L., 2014, MNRAS, 440, 75

Bartolo N., Komatsu E., Matarrese S., Riotto A., 2004, Phys. Rept., 402, 103

Baugh C. M., Gaztanaga E., Efstathiou G., 1995, MNRAS, 274, 1049

Bertolami O., Martins P. J., 2000, Phys. Rev. D, 61, 064007

Bruni M., Crittenden R., Koyama K., Maartens R., Pitrou C., Wands D., 2012, Phys. Rev. D, 85, 041301

Camera S., Santos M. G., Maartens R., 2015, MNRAS, 448, 1035

Carbone C., Mena O., Verde L., 2010, J. Cosmol. Astropart. Phys., 1007, 020

Clemson T., Koyama K., Zhao G.-B., Maartens R., Valiviita J., 2012, Phys. Rev. D, 85, 043007

Costa A. A., Xu X.-D., Wang B., Ferreira E. G. M., Abdalla E., 2014, Phys. Rev. D, 89, 103531

Crooks J. L., Dunn J. O., Frampton P. H., Norton H. R., Takahashi T., 2003, Astropart. Phys., 20, 361

Cui W., Baldi M., Borgani S., 2012, MNRAS, 424, 993

Dalal N., Dore O., Huterer D., Shirokov A., 2008, Phys. Rev. D, 77, 123514

Desjacques V., Seljak U., 2010, Class. Quant. Grav., 27, 124011

Desjacques V., Seljak U., Iliev I., 2009, MNRAS, 396, 85

Desjacques V., Jeong D., Schmidt F., 2018, Phys. Rept., 733, 1

Despali G., Vegetti S., 2017, MNRAS, 469, 1997

Duniya D. G. A., Bertacca D., Maartens R., 2015, Phys. Rev. D, 91, 063530

Efstathiou G., Bond J. R., 1999, MNRAS, 304, 75

Fay S., 2016, MNRAS, 460, 1863

Fay S., Nesseris S., Perivolaropoulos L., 2007, Phys. Rev. D, 76, 063504

Fedeli C., Moscardini L., 2010, MNRAS, 405, 681

Fonseca J., Camera S., Santos M., Maartens R., 2015, ApJ, 812, L22
Gao L., White S. D. M., Jenkins A., Stoehr F., Springel V., 2004, MNRAS, 355,819

Giannantonio T., Ross A. J., Percival W. J., Crittenden R., Bacher D., Kilbinger M., Nichol R., Weller J., 2014, Phys. Rev. D, 89, 023511

Giocoli C., Tormen G., Sheth R. K., van den Bosch F. C., 2010, MNRAS, 404, 502

Giocoli C., Tormen G., Sheth R. K., 2012, MNRAS, 422, 185

Giocoli C., Marulli F., Baldi M., Moscardini L., Metcalf R. B., 2013, MNRAS, 434, 2982

Grossi M., Dolag K., Branchini E., Matarrese S., Moscardini L., 2007, MNRAS, 382, 1261

Grossi M., Verde L., Carbone C., Dolag K., Branchini E., Iannuzzi F., Matarrese S., Moscardini L., 2009, MNRAS, 398, 321

Hamaus N., Seljak U., Desjacques V., Smith R. E., Baldauf T., 2010, Phys. Rev. D, 82, 043515

Hashim M., Bertacca D., Maartens R., 2014, Phys. Rev. D, 90, 103518

Heymans C. et al., 2013, MNRAS, 432, 2433

Hildebrandt H. et al., 2017, MNRAS, 465, 1454

Howlett C., Lewis A., Hall A., Challinor A., 2012, J. Cosmol. Astropart. Phys., 1204, 027

Jeong D., Schmidt F., Hirata C. M., 2012, Phys. Rev. D, 85, 023504

Kamionkowski M., Verde L., Jimenez R., 2009, J. Cosmol. Astropart. Phys., 0901, 010

Komatsu E. et al., 2011, ApJS, 192, 18

Koyama K., Maartens R., Song Y.-S., 2009, J. Cosmol. Astropart. Phys., 0910, 017

Kunz M., 2009, Phys. Rev. D, 80, 123001

Laureijs R. et al., 2011, preprint (arXiv:1110.3193)

Leistedt B., Peiris H. V., Roth N., 2014, Phys. Rev. Lett., 113, 221301

Lewis A., Challinor A., Lasenby A., 2000, Astrophys. J., 538, 473

Liguori M., Sefusatti E., Fergusson J. R., Shellard E. P. S., 2010, Adv. Astron., 2010, 980523

LoVerde M., Smith K. M., 2011, J. Cosmol. Astropart. Phys., 1108, 003

LoVerde M., Miller A., Shandera S., Verde L., 2008, J. Cosmol. Astropart. Phys., 0804, 014

Lucchin F., Matarrese S., 1985, Phys. Rev. D, 32, 1316

Lyth D. H., Wands D., 2002, Phys. Lett. B, 524, 5

Maartens R., Abdalla F. B., Jarvis M., Santos M. G., 2015, PoS, AASKA14, 016

Maldacena J. M., 2003, J. High Energy Phys., 05, 013

Marulli F., Baldi M., Moscardini L., 2012, MNRAS, 420, 2377

Matarrese S., Verde L., 2008, ApJ, 677, L77

Matarrese S., Verde L., Jimenez R., 2000, ApJ, 541, 10

Moresco M., Marulli F., Baldi M., Moscardini L., Cimatti A., 2014 MNRAS, 443, 2874

Moroi T., Takahashi T., 2001, Phys. Lett. B, 522, 215

Neyrinck M. C., 2008, MNRAS, 386, 2101

Neyrinck M. C., Yang L. F., 2013, MNRAS, 433, 1628

Oppizzi F., Liguori M., Renzi A., Arroja F., Bartolo N., 2018, J. Cosmol. Astropart. Phys., 1805, 045

Padilla A., 2015, preprint (arXiv: 1502.05296)

Pettorino V., Baccigalupi C., 2008, Phys. Rev. D, 77, 103003

Pillepich A., Porciani C., Hahn O., 2010, MNRAS, 402, 191

Platen E., van de Weygaert R., Jones B. J. T., 2007, MNRAS, 380, 551

Pollina G., Baldi M., Marulli F., Moscardini L., 2016, MNRAS, 455, 3075

Pollina G., Hamaus N., Dolag K., Weller J., Baldi M., Moscardini L., 2017, MNRAS, 469, 787

Pourtsidou A., Skordis C., Copeland E. J., 2013, Phys. Rev. D, 88, 083505

Raccanelli A., Bertacca D., Doré O., Maartens R., 2014, J. Cosmol. Astropart. Phys., 1408, 022

Raccanelli A., Bertacca D., Jeong D., Neyrinck M. C., Szalay A. S., 2018, Phys. Dark Univ., 19, 109

Renaux-Petel S., 2015, Comptes Rendus Physique, 16, 969

Ross A. J. et al., 2013, MNRAS, 428, 1116

Salvatelli V., Marchini A., Lopez-Honorez L., Mena O., 2013, Phys. Rev. D, 88, 023531

Salvatelli V., Said N., Bruni M., Melchiorri A., Wands D., 2014, Phys. Rev. Lett., 113, 181301 
Sartoris B. et al., 2016, MNRAS, 459, 1764

Scoccimarro R., Hui L., Manera M., Chan K. C., 2012, Phys. Rev. D, 85, 083002

Sefusatti E., Liguori M., Yadav A. P. S., Jackson M. G., Pajer E., 2009, J. Cosmol. Astropart. Phys., 0912, 022

Setare M. R., Mohammadipour N., 2013, J. Cosmol. Astropart. Phys., 1301, 015

Simpson F. et al., 2016, Phys. Rev. D, 93, 023525

Smith R. E., Scoccimarro R., Sheth R. K., 2007, Phys. Rev. D, 75, 063512

Springel V., 2005, MNRAS, 364, 1105

Springel V., White S. D. M., Tormen G., Kauffmann G., 2001, MNRAS, 328,726

Springel V. et al., 2008, MNRAS, 391, 1685

Sutter P. M., Elahi P., Falck B., Onions J., Hamaus N., Knebe A., Srisawat C., Schneider A., 2014, MNRAS, 445, 1235

Sutter P. M. et al., 2015, Astron. Comput., 9, 1

Tereno I., Doré O., van Waerbeke L., Mellier Y., 2005, A\&A, 429, 383

Väliviita J., Palmgren E., 2015, J. Cosmol. Astropart. Phys., 1507, 015
Vikhlinin A. et al., 2009, ApJ, 692, 1060

Villaescusa-Navarro F., Marulli F., Viel M., Branchini E., Castorina E., Sefusatti E., Saito S., 2014, J. Cosmol. Astropart. Phys., 1403, 011

Wagner C., Verde L., 2012, J. Cosmol. Astropart. Phys., 1203, 002

Wagner C., Verde L., Boubekeur L., 2010, J. Cosmol. Astropart. Phys., 1010, 022

Wei H., Zhang S. N., 2008, Phys. Rev. D, 78, 023011

Wei H., Liu J., Chen Z.-C., Yan X.-P., 2013, Phys. Rev. D, 88, 043510

Weinberg S., 1989, Rev. Mod. Phys., 61, 1

Wetterich C., 1988, Nucl. Phys., B302, 668

Wetterich C., 1995, A\&A, 301, 321

Zeldovich Ya. B., 1970, A\&A, 5, 84

Zhao D. H., Jing Y. P., Mo H. J., Börner G., 2009, ApJ, 707, 354

This paper has been typeset from a $\mathrm{T}_{\mathrm{E}} \mathrm{X} / \mathrm{LAT} \mathrm{E}$ file prepared by the author. 